\title{
IMPROVING GOOVAERTS' AND DE VYLDER'S STABLE RECURSIVE ALGORITHM
}

\author{
By Colin M. Ramsay
}

University of Nebraska-Lincoln

\begin{abstract}
GoovaerTs and DE VyLDER (1983) provided a stable recursive algorithm for calculating the probability of ultimate ruin. Their algorithm yielded bounds for this probability. It is shown that in practice their method may be inherently unstable because it is based on the subtraction of nearly equal numbers. An alternative to this type of subtraction is provided. It is proved that their algorithm converges only at a linear rate to the true value. It is suggested that this slow rate of convergence be improved via an application of the Richardson extrapolation technique.
\end{abstract}

\section{KEYWORDS}

Ruin probability; rounding errors; truncation errors; order of convergence; Richardson extrapolation.

\section{INTRODUCTION}

When claims follow a compound Poisson process with rate $\lambda$ and premiums are paid continuously at rate $c$, GeRBER $(1979$, p. 115 , equation (3.7)) proved that the infinite time probability of ruin for an initial risk reserve of $u, \psi(u)$, satisfied the following Volterra integral equation of the second kind:

$$
\psi(u)=\frac{\lambda}{c} \int_{u}^{\infty}(1-F(y)) d y+\frac{\lambda}{c} \int_{0}^{u} \psi(u-y)(1-F(y)) d y,
$$

where $c=\lambda p_{1}(1+\theta)$ is the premium rate, $p_{1}$ is the expected claim size, $\theta>0$ is the loading and $F(x)$ is the cumulative distribution function (cdf) of the claim size random variable. $F(x)$ is assumed to be completely known.

Techniques for numerically solving equation (1) are contained in several texts including BaKer (1977), Delves and Mohamed (1985), and BrunNer and VAN DER HOUWEN (1986). However they do not provide methods for obtaining tight bounds on the true solution $\psi(u)$.

GoOvAERTS and DE VyLDER (1983) developed a recursive algorithm for approximating $\psi(u)$ and for providing bounds on their approximations. Their 
method can be summarized as follows (with a change in notation): let

$$
K(s)=\int_{s}^{\infty} \frac{(-F(y))}{p_{1}} d y, \quad s \geq 0 .
$$

Here $K(s)$ has been standardized to ensure that $K(0)=1$. For fixed $u$, the step size $h$ and the number of steps $n$ are defined to satisfy $n h=u$. The bounds of $\psi(u)$ are then calculated recursively as follows: for $j=0,1,2, \ldots, n$

$$
h=\frac{u}{n}
$$

$$
\psi(0)=\frac{1}{1+\theta}
$$

$$
\begin{aligned}
& \hat{\psi}_{u}(j h)=\frac{1}{(1+\theta)}\left[K(j h)-\sum_{i=1}^{j} \hat{\psi}_{u}((j-i) h) \Delta K((i-1) h)\right] \\
& \hat{\psi}_{l}(j h)=\frac{\left[K(j h)-\sum_{i=1}^{j-1} \hat{\psi}_{l}((j-i) h) \Delta K(i h)\right]}{[1+\theta+\Delta K(0)]}
\end{aligned}
$$

where $\hat{\psi}_{u}(x)$ and $\hat{\psi}_{l}(x)$ are the upper and lower bounds respectively on the true value of $\psi(x), x>0$, and $\Delta$ is the well known forward difference operator defined with respect to step size $h$, i.e., for any real valued function $g(y)$,

$$
\Delta g(y)=g(y+h)-g(y) \text {. }
$$

The resulting approximation to $\psi(u)$ is

$$
\hat{\psi}(u) \approx \frac{1}{2}\left[\hat{\psi}_{u}(n h)+\hat{\psi}_{l}(n h)\right] .
$$

with an upper bound on the error given by

$$
|\hat{\psi}(u)-\psi(u)| \leq \hat{\psi}_{u}(n h)-\hat{\psi}_{l}(n h) .
$$

For fixed $h$ and $u$, Goovaerts and de Vylder proved that there is no cumulative effect of the propagation of errors in equations (4) and (5). In particular, if $\varepsilon_{i}$ is the absolute value of the error in $\hat{\psi}(i h), i=1,2,3, \ldots, j-1$, then the absolute error in $\hat{\psi}(j h)$ is $\varepsilon_{j}$ satisfying

$$
\varepsilon_{j} \leq \operatorname{Max}\left\{\varepsilon_{1}, \varepsilon_{2}, \ldots, \varepsilon_{j-1}\right\} .
$$

Since this is true for $j=2,3, \ldots$, then

$$
\varepsilon_{j} \leq \varepsilon_{1} \quad j=1,2, \ldots, n .
$$

The classic reference on the analysis of the propagation of errors in a series of calculations is WILKINSON (1963). 
The objective of this paper is to improve the practical implementation of equations (4) and (5) so as to reduced the sequence of errors $\left\{\varepsilon_{j}\right\}$. Numerical results are given for the Pareto distribution.

In the sequel, it will always be assumed that $F(x)$ is differentiable and yields the probability distribution function (pdf) $f(y)$. For most of the distributions used by actuaries, the pdf $f(x)$ is infinitely differentiable except, perhaps, at a countable number of points.

\section{MAIN RESULTS}

\subsection{Rounding errors}

Goovaerts and de Vylder's strategy for approximating $\psi(u)$ was to use equations (4) and (5) for successively smaller values of $h$ (i.e., larger values of $n$ ), stopping when they obtained agreement to some desired degree of accuracy. RALSTON and RABINOWITZ (1978, chapter 4.2, p. 93) pointed out that, when using floating arithmetic, this procedure is fraught with danger since rounding errors will eventually dominate the calculations. This is because, as $h \rightarrow 0$, the difference between $K((i-1) h)$ and $K(i h)$ tends to the difference of two nearly equal numbers, and thus contains fewer and fewer significant digits. They recommended that $h$ should not be too small and that the Richardson extrapolation technique be used to improve the accuracy of the approximations.

To reduce the loss of significant digits, one must avoid subtractions, especially subtracting nearly equal terms. For some distributions, it is possible to calculate this difference "exactly". For example, in the Pareto case where $F(x)=1-1 /(1+x)^{2}$ and $K(x)=1 /(1+x)$, it is better to calculate $\Delta K(i h)$ as $-h /[(1+i h)(1+(i+1) h)]$ rather than as $[1 /(1+(i+1) h)]-[1 /(1+i h)]$.

Unfortunately, there are distributions where neither $F(x)$ nor $K(x)$ can be obtained exactly in closed form. However, for most of these distributions, $f(y)$ can be calculated "exactly". In such cases $\Delta K(x)$ must be evaluated very carefully because both $K(x)$ and $F(x)$ may be known only to small number of decimal places or significant. When $h$ is small, it is better to compute $\Delta K(x)$ as follows:

$$
\begin{aligned}
\Delta K(x) & =-\frac{1}{p_{1}} \int_{x}^{x+h}(1-F(y)) d y \\
& =-\frac{1}{p_{1}}\left[h-\int_{x}^{x+h} F(y) d y\right] \\
& =-\frac{1}{p_{1}}\left[h-((x+h) F(x+h)-x F(x))+\int_{x}^{x+h} y f(y) d y\right]
\end{aligned}
$$

The integral terms in equations (7) and (8) may have to be evaluated numerically using a composite Gauss-Legendre or a composite Newton-Cotes 
quadrature formula. These formulas do not involve subtractions-see Ralston and Rabinowitz (chapter 4) and as such may result in the loss of at most 1 or 2 significant digits.

Equation (8) is recommend over equation (7) because (8) requires fewer (only 2) evaluations of $F(x)$ hence should require less time to calculate $\Delta K(x)$. Since $f(y)$ can be obtained exactly, $F(x)$ should be calculated to a large number of significant digits. This will ensure that $\Delta x F(x)=$ $(x+h) F(x+h)-x F(x)$ can be evaluated accurately. Note $\Delta x F(x)$ represents the subtraction of nearly equal terms and, if not evaluated carefully, may result in a significant loss of significant digits. The integral term in equation (8) can be obtained to any practical degree of accuracy.

\subsection{Truncated error}

An important aspect in the development of equations (4) and (5) is Goovaerts and de Vylder's discretization of their integrals. This requires implicit use of the following composite quadrature rule:

$$
\int_{0}^{x_{j}} \psi\left(x_{j}-s\right) d K(s) \approx \sum_{i=1}^{j} \psi((j-i) h) \Delta K((i-1) h)
$$

where $h=u / n$ and $x_{j}=j h$. Unfortunately this rule yields exact results if, and only if, $K(x)$ is a constant or a step function with discontinuities at $h, 2 h, \ldots j h$. It is therefore not as accurate as more traditional rules such as the composite trapezoid of Simpson's rules. However it does provide upper and lower bounds on $\psi(x)$.

It is well known that the trapezoid rule has a truncation error of $O\left(h^{2}\right)$ while the Simpson's rule has an error of $O\left(h^{4}\right)$. What is the order of the error in equation (9)? It will be proved that the error in equation (9) is $O(h)$. To establish this result one needs the Euler-Maclaurin Summation Formula (Ralston and Rabinowitz, page 138):

Result 1 (Euler-Maclaurin). If $g(x)$ has derivatives of order $2 m+2$, then for $r$ a positive integer and $h>0$,

$$
\begin{aligned}
h \sum_{i=0}^{r} g\left(y_{0}+i h\right)= & \int_{y_{0}}^{y_{0}+r h} g(s) d s+\frac{h}{2}\left[g\left(y_{0}+r h\right)-g\left(y_{0}\right)\right] \\
& +\sum_{k=1}^{m} \frac{B_{2 k} h^{2 k}}{(2 k) !}\left[g^{(2 k-1)}\left(y_{0}+r h\right)-g^{(2 k-1)}\left(y_{0}\right)\right]+E_{m}
\end{aligned}
$$

where $g^{(i)}(s)$ is the $i$-th derivative of $g(s)$,

$$
E_{m}=\frac{r h^{2 m+2} B_{2 m+2}}{(2 m+2) !} g^{(2 m+2)}(\xi), \quad y_{0}<\xi<y_{0}+r h
$$


IMPROVING GOOVAERTS' AND DE VYLDER'S STABLE RECURSIVE ALGORITHM 55

and the $B_{k}$ 's are Bernoulli numbers, i.e.,

$$
\frac{t}{e^{t}-1} \equiv \sum_{k=1}^{\infty} B_{k} \frac{t^{k}}{k !} \text {. }
$$

Theorem 1. Assume:

(1) the pdf $f(y)$ is bounded by $M$,

(2) $x>0$ is fixed, and

(3) $r$ is a positive integer such that $x=r h$.

As $h \rightarrow 0$, the truncation error $E$, defined by

$$
E=\sum_{i=1}^{r} \psi((r-i) h) \Delta K((i-1) h)-\int_{0}^{x} \psi(x-s) d K(s),
$$

is of $\operatorname{order} h$, i.e., $E=O(h)$.

Proof : Using equation (10), let $y_{0}=0$ and replace $r h$ by $x$. Since $x$ is held to be constant, equation (10) can be rearranged to give

$$
h \sum_{i=1}^{r} g(i h)-\int_{0}^{x} g(s) d s=a_{0} h+\sum_{k=1}^{m} a_{2 k} h^{2 k}+a_{2 m+1} h^{2 m+1}
$$

where the $a_{k}$ 's are constants depending on the $B_{k}$ 's, the $g^{(k)}(x)$ 's and the $g^{(k)}(0)$ 's. Note the summation on the left starts from $i=1$. The final term in equation (12) is $h^{2 m+1}$ rather than $h^{2 m+2}$ because in the $E_{m}$ term (in equation (10)) $r h=x$, a constant.

Assume $g(s)$, given by

$$
g(s)=\psi(x-s) K^{(1)}(s),
$$

is at least twice differentiable, i.e., $m \geq 0$. Since $K(s)$ must be at least twice differentiable, then

$$
\Delta K((i-1) h)=h K^{(1)}(i h)-\frac{h^{2}}{2} K^{(2)}\left(\xi_{i}\right)
$$

where $(i-1) h<\xi_{i}<i h$. So equation (11) implies

$$
\begin{aligned}
E= & \sum_{i=1}^{r} \psi((r-i) h) \Delta K((i-1) h)-\int_{0}^{x} \psi(x-s) d K(s) \\
= & h \sum_{i=1}^{r} \psi(x-i h) K^{(1)}(i h)-\int_{0}^{x} \psi(x-s) K^{(1)}(s) d s- \\
& -\frac{h^{2}}{2} \sum_{i=1}^{r} \psi((r-i) h) K^{(2)}\left(\xi_{i}\right) \\
= & -\frac{h^{2}}{2} \sum_{i=1}^{r} \psi((r-i) h) K^{(2)}\left(\xi_{i}\right)+a_{0} h+\sum_{k=1}^{m} a_{2 k} h^{2 k}+a_{2 m+1} h^{2 m+1} .
\end{aligned}
$$


Since $f(s)$ is bounded by $M, K^{(2)}(s)=f(s) / p_{1}$ and $0 \leq \psi(s) \leq 1$, it follows that

$$
\frac{h^{2}}{2} \sum_{i=1}^{r} \psi(x-i h) K^{(2)}\left(\xi_{i}\right) \leq \frac{h^{2} r M}{2 p_{1}}=\frac{x h M}{2 p_{1}}=O(h) .
$$

It follows that, for $m \geq 0$, the terms on the right hand side of equation (13) yield

$$
E=O(h)+a_{0} h+O\left(h^{2}\right)+O\left(h^{2 m+1}\right)=O(h) .
$$

Q.E.D.

This suggests that for fixed $u$, as $h \rightarrow 0$ (or $n \rightarrow \infty$ ), the approximation in equation (6) may converge slowly $(O(h))$ to the true value $\psi(u)$. This means that one must use very small values of $h$ (very large values of $n$ ) or find a way to accelerate this slow rate of convergence. However, from Section 2.1 above, the use very small values of $h$ was not recommended and it was suggested that an acceleration technique be used. This approach will now be investigated.

\subsection{Accelerating convergence}

Assume that as $h \rightarrow 0$, the error $E$, in equations (11) and (14), can be written as

$$
E=\beta_{1}^{(0)} h+\sum_{k=2}^{\infty} \beta_{k}^{(0)} h^{k}
$$

where the constants $\beta_{k}^{(0)}, k=1,2, \ldots$, need not be known. One can use the Richardson extrapolation technique (Ralston and Rabinowitz, page 94) to accelerate the convergence of the sequence of approximations $\{\hat{\psi}(x ; i)\}$ where $\hat{\psi}(x ; i)$ is calculated using a step-size $h_{i}=x / n_{i}$. The true value $\psi(x)$ is given by

$$
\psi(x)=\hat{\psi}(x ; i)+\beta_{1}^{(0)} h_{i}+\sum_{k=2}^{\infty} \beta_{k}^{(0)} h_{i}^{k} .
$$

If $\hat{\psi}(x ; i)$ is calculated for two step sizes $h_{1}$ and $h_{2}$, with $h_{2}=\rho h_{1}$, $0<\rho<1$, then $\beta_{0}^{(0)}$ can be eliminated from equation (15) to give

$$
\psi(x)=\hat{\psi}(x ; 1,2)+\sum_{k=2}^{\infty} \beta_{k}^{(1)} h_{1}^{k}
$$

where

$$
\hat{\psi}(x ; 1,2)=\frac{\hat{\psi}(x ; 2)-\rho \hat{\psi}(x ; 1)}{1-\rho}
$$


and the $\beta_{k}^{(1)}$ are constants depending on $\beta_{k}^{(0)}$ and $\rho$. Thus the new approximation is $\hat{\psi}(x ; 1,2)$ which has an error of order $h_{1}^{2}$. If one wanted to eliminate the $h_{1}^{2}$ term in $(16)$ then use $\hat{\psi}(x ; 1,2)$ and $\hat{\psi}(x ; 2,3)$ :

$$
\psi(x)=\hat{\psi}(x ; 1,2,3)+\sum_{k=3}^{\infty} \beta_{k}^{(2)} h_{1}^{k}
$$

where

$$
\hat{\psi}(x ; 1,2,3)=\frac{\hat{\psi}(x ; 2,3)-\rho^{2} \hat{\psi}(x ; 1,2)}{1-\rho^{2}} .
$$

The error is now of order $h_{1}^{3}$.

This process can be repeatedly applied as follows: following the notation of Ralston and Rabinowitz, let $\hat{\psi}(x ; i)$ be denoted by $T_{0}^{i}, i=1,2, \ldots$ A lower triangular matric of approximations $T_{r}^{i}$ can be generated as follows: for $r=1,2,3, \ldots$,

$$
T_{r}^{i+1}=\frac{T_{r-1}^{i+1}-\rho^{r} T_{r-1}^{i}}{1-\rho^{r}}=T_{r-1}^{i+1}+\frac{T_{r-1}^{i+1}-T_{r-1}^{i}}{\rho^{-\mathrm{r}}-1}
$$

where $h_{i+1}=\rho h_{i}$ and $\rho<1$. If the step sizes do not decrease uniformly, then simply replace $\rho^{r}$ by $h_{i+r} / h_{i}$. The element $T_{r}^{r-1}$ is actually the approximation $\hat{\psi}(x ; 1,2 \ldots r)$; it appears in the lower right hand corner of the matrix.

This procedure can be applied to both the upper and lower bounds approximations. The final approximation to $\psi(x)$ will be

$$
\psi(x) \approx \frac{1}{2}\left[\hat{\psi}_{u}(x ; 1,2, \ldots, r)+\hat{\psi}_{l}(x ; 1,2, \ldots, r)\right]
$$

where the subscripts $u$ and $l$ refer to upper and lower bounds respectively.

Warning 1. It may not always be the case that the extrapolation procedure $T_{r}^{i}$ will retain the upper and lower bound properties, i.e.,

$$
\hat{\psi}_{l}(x ; 1,2, \ldots, r) \leq \psi(x) \leq \hat{\psi}_{u}(x ; 1,2, \ldots, r)
$$

may be violated.

\section{NUMERICAL RESULTS}

Using the Pareto distribution used by Goovaerts and de Vylder, ruin probabilities are approximated for $\theta=0.2$ and $u=10,50$ and 100 as follows:

1. using double precision arithmetic;

2. using 5 step sizes: $h=2^{-k}, k=1,2 \ldots 5$; 
3. carefully evaluating the difference $K((i-1) h)-K(i h)$ according to equation (8);

4. calculating $\hat{\psi}_{u}(u)$ and $\hat{\psi}_{l}(u)$ according to equations (4) and (5);

5. accelerating the convergence of the upper and lower bounds sequences using equation (17); then

6. calculating the final estimates using equation (18).

The procedure outlined above will now be used to calculate the probability of ruin for the Pareto

$$
F(x)=1-(1+x)^{-2}
$$

taken from Goovaerts and de Vylder. These results are contained in Tables 1 to 3. The extrapolation procedure vastly improves the accuracy of the initial approximations given by the $T_{0}^{i}$, s. From the final results shown in Table 3 , it is clear that the estimate $\hat{\psi}(u)$ is accurate to at least 7 decimal places. In fact, even if one had used only the first three rows (i.e., $h=0.5,0.25,0.125$ ) for the extrapolation procedure, the resulting approximation based on $T_{2}^{3}$ would be accurate to at least 4 decimal places. This is more accurate than $T_{0}^{5}$ and requires less computations! Note that in Tables 1 an $2, T_{0}^{5}$ is accurate to 2 or 3 decimal places.

Finally, for $u=10.0$, the lower bounds provided by Goovaerts and de Vylder do not agree with mine. I cannot explain this difference.

TABLE 1

Richardson's Extrapolation of LowEr Bounds, $\theta=0.20$

\begin{tabular}{c|r|c|ccccc}
\hline \hline$u$ & $n$ & $i$ & $\hat{\psi}_{1}(u)=T_{0}^{i}$ & $T_{1}^{i}$ & $T_{2}^{i}$ & $T_{3}^{i}$ & $T_{4}^{i}$ \\
\hline 10.0 & 20 & 1 & 0.41761640 & & & & \\
10.0 & 40 & 2 & 0.42596352 & 0.43431064 & & & \\
10.0 & 80 & 3 & 0.43042938 & 0.43489525 & 0.43509011 & & \\
10.0 & 160 & 4 & 0.43273608 & 0.43504277 & 0.43509194 & 0.43509221 & \\
10.0 & 320 & 5 & 0.43390772 & 0.43507937 & 0.43509157 & 0.43509152 & 0.43509148 \\
\hline 50.0 & 100 & 1 & 0.13805696 & & & & \\
50.0 & 200 & 2 & 0.14079460 & 0.14353224 & & & \\
50.0 & 400 & 3 & 0.14228559 & 0.14377659 & 0.14385804 & & \\
50.0 & 800 & 4 & 0.14306363 & 0.14384166 & 0.14386335 & 0.14386411 & \\
50.0 & 1600 & 5 & 0.14346099 & 0.14385835 & 0.14386391 & 0.14386399 & 0.14386398 \\
\hline 100.0 & 200 & 1 & 0.06716234 & & & & \\
100.0 & 400 & 2 & 0.06809997 & 0.06903759 & & & \\
100.0 & 800 & 3 & 0.06861110 & 0.06912223 & 0.06915045 & & \\
100.0 & 1600 & 4 & 0.06887802 & 0.06914494 & 0.06915250 & 0.06915280 & \\
100.0 & 3200 & 5 & 0.06901440 & 0.06915078 & 0.06915273 & 0.06915277 & 0.06915276 \\
\hline
\end{tabular}


TABLE 2

RichARDSON'S EXTRAPOLATION OF UPPER BOUNDS, $\theta=0.20$

\begin{tabular}{c|c|c|ccccc}
\hline \hline$u$ & $n$ & $i$ & $\hat{\psi}_{u}(u)=T_{0}^{i}$ & $T_{1}^{i}$ & $T_{2}^{i}$ & $T_{3}^{i}$ & $T_{4}^{i}$ \\
\hline 10.0 & 20 & 1 & 0.45552952 & & & & \\
10.0 & 40 & 2 & 0.44497968 & 0.43442983 & & & \\
10.0 & 80 & 3 & 0.43994494 & 0.43491021 & 0.43507033 & & \\
10.0 & 160 & 4 & 0.43749479 & 0.43504464 & 0.43508945 & 0.43509219 & \\
10.0 & 320 & 5 & 0.43628720 & 0.43507961 & 0.43509126 & 0.43509152 & 0.43509148 \\
\hline 50.0 & 100 & 1 & 0.15110109 & & & & \\
50.0 & 200 & 2 & 0.14729514 & 0.14348918 & & & \\
50.0 & 400 & 3 & 0.14553310 & 0.14377106 & 0.14386501 & & \\
50.0 & 800 & 4 & 0.14468703 & 0.14384096 & 0.14386427 & 0.14386416 & \\
50.0 & 1600 & 5 & 0.14427264 & 0.14385826 & 0.14386402 & 0.14386399 & 0.14386398 \\
\hline 100.0 & 200 & 1 & 0.07164847 & & & & \\
100.0 & 400 & 2 & 0.07033307 & 0.06901767 & & & \\
100.0 & 800 & 3 & 0.06972639 & 0.06911970 & 0.06915371 & & \\
100.0 & 1600 & 4 & 0.06943550 & 0.06914462 & 0.06915293 & 0.06915281 & \\
100.0 & 3200 & 5 & 0.06929312 & 0.06915074 & 0.06915279 & 0.06915277 & 0.06915276 \\
\hline
\end{tabular}

TABLE 3

Final Approximation, $\theta=0.20$

\begin{tabular}{r|cc|c}
\hline \multicolumn{1}{c|}{$u$} & $\hat{\psi}_{1}(u)=T_{4}^{5}$ & $\hat{\psi}_{u}(u)=T_{4}^{5}$ & $\hat{\psi}(u)$ \\
\hline 10.0 & 0.43509148 & 0.43509148 & 0.43509148 \\
50.0 & 0.14386398 & 0.14386398 & 0.14386398 \\
100.0 & 0.06915276 & 0.06915276 & 0.06915276 \\
\hline
\end{tabular}

\section{REFERENCES}

Abramowitz, M. and Stegun, I. A. (1972) Handbook of Mathematical Functions, 9th edition. Dover Publications, New York.

BAKER, C.T.H. (1977) The Numerical Treatment of Integral Equations. Clarendo Press, Oxford. Brunner, H. and van der Houwen, P. J. (1986) The Numerical Solution of Volterra Equations. North Holland, Amsterdam.

Delves, L. M. and Mohamed, J. L. (1985) Computational Methods for Integral Equations. University Press, Cambridge.

Gerber, H. U. (1979) An Introduction to Mathematical Risk Theory. Huebner Foundation Monograph, University of Pennsylvania, Philadelphia.

Goovaerts, M. and DE Vylder, F. (1983) A Stable Recursive Algorithm for Evaluation of Ultimate Ruin Probabilities. ASTIN Bulletin 14, 53-59.

Ralston, A. and Rabinowitz, P. (1978) A first Course in Numerical Analysis, 2nd edition. International Student Edition, McGraw-Hill, Japan.

THORIN, O. and WIKSTAD, N. (1977) Calculation of ruin probabilities when the claim distribution is lognormal. ASTIN Bulletin 9, 231-246.

Wilkinson, J.H. (1963) Rounding Errors in Algebra Processes. Prentice-Hall, New Jersey.

\section{COLIN M. RAMSAY}

Actuarial Science, 310 Burnett Hall, University of Nebraska-Lincoln, Lincoln, NE 68588-0307, U.S.A. 